\title{
Evaluation of a Sliding Mode Fault Tolerant Controller on the MuPAL- $\alpha$ Research Aircraft
}

\author{
L. Chen ${ }^{1}$, H. Alwi ${ }^{1}$, C. Edwards ${ }^{1}$ and M. Sato ${ }^{2}$
}

\begin{abstract}
This paper presents piloted flight test results of a sliding mode fault tolerant control scheme implemented on the Japan Aerospace Exploration Agency's MuPAL- $\alpha$ research aircraft. These results represent unique piloted validation tests of a sliding mode fault tolerant control allocation scheme on a full-scale aircraft operating in the presence of actuator faults. The control law used here does not require the presence of a fault detection and isolation unit and therefore in the event of faults/failures, the actuator effectiveness levels are unknown. In the absence of this information, a fixed control allocation mechanism has been used in order to retain nominal fault-free performance. The control scheme has been implemented on the lateral-directional motion and incorporated within the experimental fly-by-wire system. Piloted flight test results show that close to nominal tracking performance can be maintained despite the presence of unknown actuator faults as well as actuator uncertainties.
\end{abstract}

\section{INTRODUCTION}

Fault Detection and Diagnosis (FDD), and Fault Tolerant Control (FTC) [1] methodologies, have been identified as an effective way to improve the resilience and tolerance of systems to faults. Such ideas received significant attention from the aerospace industry: in particular aircraft manufacturers have investigated advanced FDD/FTC approaches for aircraft Guidance, Navigation and Control (GNC). Some representative EU funded projects, such as GARTEUR FM-AG16 [2], ADDSAFE [3], RECONFIGURE [4], [5] and VISION [6], [7], have sought to increase the Technology Readiness Level (TRL) of modern advanced FDD/FTC approaches (see Fig. 1). The Japan Aerospace Exploration Agency (JAXA) has also flight tested restructurable flight control systems and real-time flight trajectory optimisation systems [8], [9], [10]. The ongoing EU/Japan co-funded project VISION exploits JAXA's Multi-Purpose Aviation Laboratory (MuPAL- $\alpha$ ) research aircraft [11], [12] and seeks to validate advanced FDD and FTC schemes in piloted flight tests. As part of VISION, adaptive

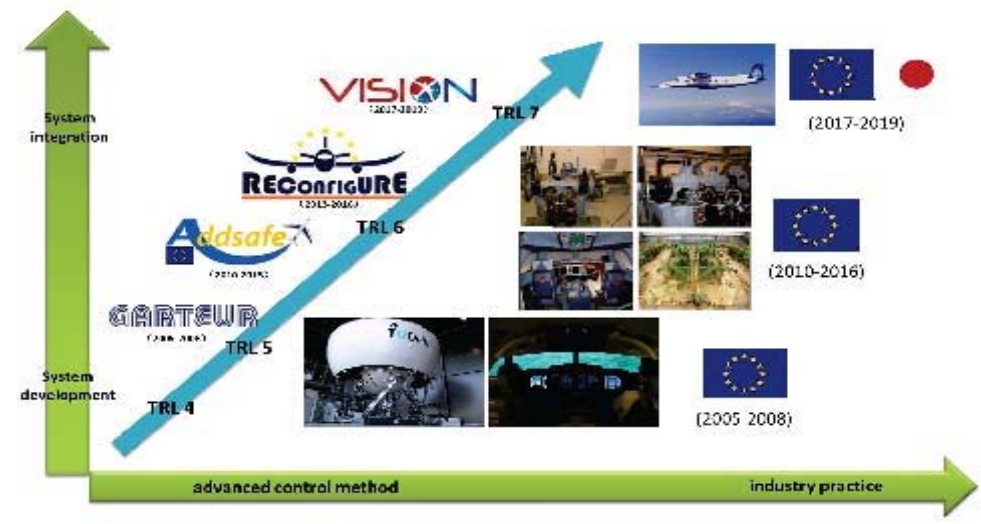

Fig. 1. EU projects

[13], $H_{\infty}$ [14] and sliding mode methods [6] have been tested on a Hardware-in-the-Loop (HIL) platform as a precursor to piloted flight tests.

One thread of the FTC literature has considered sliding mode schemes in conjunction with a simple form of control allocation [15]. An example of this class of controller has been successfully integrated within the MuPAL- $\alpha$ Fly-By-Wire (FBW) system to cope with actuator faults/failures and subjected to both HIL and piloted flight tests [6], [7]. The validation results in [6] were based on ground tests, and the work in [7] validated an on-line control allocation method which requires faults/failures information to be available.

In this paper, a sliding mode fixed Control Allocation (CA) scheme is implemented within MuPAL- $\alpha$ 's FBW system and then flight tested. The proposed scheme exploits the robustness properties induced by Sliding Mode Control (SMC) to reduce the effect of uncertainty and faults/failures, and a fixed CA structure that fully utilises the available redundancy within the system and distributes the control signals to the actuators, without knowledge of the faults/failures. This control scheme is therefore much simpler than the one flight tested in [7] and does not require an FDI scheme. The controller in this paper is

\footnotetext{
${ }^{1}$ College of Engineering, Mathematics and Physical Sciences, University of Exeter, EX4 4QF, UK. 1c427@exeter.ac.uk, h.alwi@exeter.ac.uk, C.Edwards@exeter.ac.uk

2 Japan Aerospace Exploration Agency, Mitaka, Tokyo 181-0015, Japan. sato.masayuki@jaxa.jp
} 
developed within a Linear Parameter Varying (LPV) framework. This allows robust performance of the closed-loop scheme to be ensured over a wide range of flight conditions [16], [17].

This paper focusses on lateral-directional control, and considers fault scenarios wherein the ailerons and rudder are assumed to operate at reduced effectiveness levels. The objective of the paper is to develop FTC schemes that can retain close to nominal fault free performance despite actuator faults/failures. The main contribution of the paper is it describes the first piloted flight validation of a sliding mode fixed control allocation scheme.

\section{LPV SLIDING MODE CONTROLLER DESIGN}

\section{A. Preliminaries}

In this section, an LPV sliding mode FTC scheme with fixed CA is developed. Consider an LPV system with actuator faults/failures modelled as

$$
\dot{x}_{p}(t)=A_{p}(\rho) x_{p}(t)+B_{p}(\rho)\left(I_{m}-K(t)\right) u(t)
$$

where $A(\rho) \in \mathbb{R}^{n \times n}$ and $B(\rho) \in \mathbb{R}^{n \times m}$. The state vector is denoted by $x_{p} \in \mathbb{R}^{n}$ and the control input is denoted by $u_{p} \in \mathbb{R}^{m}$. Here it is assumed the state is available for control design. The time varying parameter $\rho \in \mathbb{R}^{n_{r}}$ belongs to a polytope $\Omega \subset \mathbb{R}^{n_{r}}$ and is assumed to be measurable. Here it is assumed $A(\rho)$ depends affinely on $\rho(t)$, i.e.

$$
A(\rho)=A_{0}+A_{1} \rho_{1}(t) \ldots+A_{n_{r}} \rho_{n_{r}}(t)
$$

where the $A_{i} \in \mathbb{R}^{n \times n}$ for $i=0 \ldots n_{r}$ are fixed matrices. In (1), the weighting matrix $K(t)$ is defined as

$$
K(t):=\operatorname{diag}\left(k_{1}(t), \ldots, k_{m}(t)\right)
$$

where the $k_{i}(t) \in\left[\begin{array}{ll}0 & 1\end{array}\right]$, for $i=1 \ldots m$, are time varying scalars which model the loss of effectiveness of the actuators [15]. For a fault-free actuator $k_{j}(t)=0$ and for a failed actuator $k_{j}(t)=1$. When $0<k_{j}(t)<1$, the actuator is affected by the fault and behaves with reduced effectiveness.

Let the signal $y_{c}$ represent controlled outputs

$$
y_{c}(t)=C_{c} x_{p}(t)
$$

where $C_{c} \in \mathbb{R}^{l \times n}$. Here it is assumed that the system is over-actuated and $l \leq m$. This redundancy will subsequently be exploited by a control allocation mechanism [15].

To introduce tracking, define integrator states as

$$
\dot{x}_{r}(t)=r(t)-C_{c} x_{p}(t)
$$

where $r(t) \in \mathbb{R}^{l}$ represents the differentiable command signal for the controlled outputs. It is assumed $r(t)$ satisfies

$$
\dot{r}=\Gamma\left(r(t)-R_{c}\right)
$$

where $R_{c}$ represents a fixed demand vector and $\Gamma \in \mathbb{R}^{l \times l}$ is a stable design matrix. The system in (5) represents a low-pass pre-filter and $\Gamma$ can be viewed as a design parameter.

Define $x=\operatorname{col}\left(x_{r}, x_{p}\right)$ and create an augmented system

$$
\dot{x}(t)=\underbrace{\left[\begin{array}{cc}
0 & -C_{c} \\
0 & A_{p}(\rho)
\end{array}\right]}_{A(\rho)} x(t)+\underbrace{\left[\begin{array}{c}
0 \\
B_{p}(\rho)
\end{array}\right]}_{B(\rho)}(I-K(t)) u(t)+\underbrace{\left[\begin{array}{c}
I_{l} \\
0
\end{array}\right]}_{B_{c}} r(t)
$$

In this paper, it is assumed that the time varying matrix $B(\rho)$ in (6) can be factorized as

$$
B(\rho)=B_{v} B_{2}(\rho)
$$

where $B_{v} \in \mathbb{R}^{(n+l) \times l}$ is a fixed matrix, and $B_{2}(\rho) \in \mathbb{R}^{l \times m}$ is a matrix with varying components depending on the scheduling parameter.

Remark 2.1: The ramifications of this type of factorisation are described in [18]. This is also a feature of certain (classical) control allocation papers [19].

Assumption 2.1: Assume $\operatorname{rank}\left(B_{2}(\rho)\right)=l$ for all $\rho \in \Omega$.

Assumption 2.2: The pair $\left(A(\rho), B_{v}\right)$ is controllable and $\operatorname{rank}\left(B_{v}\right)=l$. form

Since by assumption $\operatorname{rank}\left(B_{v}\right)=l$, there exists an orthogonal coordinate transformation which gives $B_{v}$ the partitioned

$$
B_{v}=\left[\begin{array}{c}
0 \\
B_{v 2}
\end{array}\right]
$$


where $\operatorname{det}\left(B_{v 2}\right) \neq 0$. The partition in (8) constitutes so-called regular form [15], [20] and the transformation can be obtained using QR factorisation.

Without loss of generality, after scaling the last $l$ states, the property $B_{v 2} B_{v 2}^{T}=I_{l}$ can always be achieved and (6) can be written as

$$
\dot{x}(t)=A(\rho) x(t)+\left[\begin{array}{c}
0 \\
B_{v 2} B_{2}(\rho)(I-K(t))
\end{array}\right] u(t)+B_{c} r(t)
$$

Suppose the actual physical control signals sent to the actuators are given by

$$
u(t):=B_{2}(\rho)^{\dagger} B_{v 2}^{T} v(t)
$$

where $B_{2}(\rho)^{\dagger}=B_{2}(\rho)^{T}\left(B_{2}(\rho) B_{2}(\rho)^{T}\right)^{-1}$. The signal $v(t) \in \mathbb{R}^{l}$ represents a virtual control signal and satisfies

$$
v(t):=B_{v 2} B_{2}(\rho) u(t)
$$

As a consequence, substituting from (10), equation (9) can be written as

$$
\dot{x}(t)=A(\rho) x(t)+\left[\begin{array}{l}
0 \\
I
\end{array}\right] v-\left[\begin{array}{c}
0 \\
B_{v 2} B_{2}(\rho) K(t) B_{2}(\rho)^{\dagger} B_{v 2}^{T}
\end{array}\right] v(t)+B_{c} r(t)
$$

The virtual control $v(t)$ will be designed for (12) in the nominal case when $K(t)=0$.

\section{B. Definition of the switching function}

For all faults/failures, a virtual control law $v(t)$ is required to be calculated to ensure closed-loop stability of the system in (12) for all admissible values of $K(t)$.

Partition the states in (12) as $x=\operatorname{col}\left(x_{1}, x_{2}\right)$ and partition accordingly $A(\rho)$ and $B_{c}$ in (12) as

$$
A(\rho)=\left[\begin{array}{ll}
A_{11}(\rho) & A_{12}(\rho) \\
A_{21}(\rho) & A_{22}(\rho)
\end{array}\right], B_{c}=\left[\begin{array}{l}
B_{c 1}(\rho) \\
B_{c 2}(\rho)
\end{array}\right]
$$

where $A_{11}(\rho) \in \mathbb{R}^{n \times n}$.

Define a switching function according to [20], [21]:

$$
s(t):=S x(t)
$$

where

$$
S=\left[\begin{array}{ll}
M & I_{l}
\end{array}\right]
$$

and $M \in \mathbb{R}^{l \times n}$ represents the design degree of freedom.

During sliding $\dot{s}(t)=s(t)=0$, and the reduced order sliding motion is governed by

$$
\dot{x}_{1}(t)=\hat{A}_{11}(\rho) x_{1}(t)+B_{c 1} r(t)
$$

where $\hat{A}_{11}(\rho)=\left(A_{11}(\rho)-A_{12}(\rho) M\right)$. Since $\hat{A}_{11}(\rho)$ is dependent on $M$, the choice of $M$ may be viewed as a state feedback problem for quadratically stabilizing the pair $\left(A_{11}(\rho), A_{12}(\rho)\right)$.

\section{Control law with fixed control allocation}

In this section, a control law $v(t)$ is selected to ensure a stable sliding motion on the hyperplane $\mathcal{S}=\{x: S x=0\}$ [20], [21]. The structure of the virtual control law is

$$
v=v_{l}+v_{n}
$$

where $v_{l}$ represents a 'linear' component and $v_{n}$ is a 'nonlinear' component used to maintain sliding. Here specifically

$$
v_{l}=-S A(\rho) x(t)+\Phi s(t)-\hat{B}_{c 2} r(t)
$$

where $\hat{B}_{c 2}=S B_{c}$ and $\Phi \in \mathbb{R}^{l \times l}$ is a stable design matrix, and the nonlinear component

$$
v_{n}=-\mathcal{K}(t, x) \frac{P_{2} s(t)}{\left\|P_{2} s(t)\right\|} \quad \text { if } \quad s(t) \neq 0
$$

where the scalar function $\mathcal{K}(t, x)>0$ and the symmetric positive definite matrix $P_{2} \in \mathbb{R}^{l \times l}$ satisfies the Laypunov equation

$$
P_{2} \Phi+\Phi^{T} P_{2}=-I_{l}
$$

Consider the matrix inequality

$$
\left(B_{2}(\rho) K(t) B_{2}(\rho)^{T}\left(B_{2}(\rho)\right) K(t) B_{2}(\rho)^{T}\right)<(1-\epsilon)^{2}\left(B_{2}(\rho) B_{2}(\rho)^{T} B_{2}(\rho) B_{2}(\rho)^{T}\right)
$$


for all $\rho \in \Omega$ where $0<\epsilon<1$. Notice that if $K=0$ then (21) is satisfied. Define the admissible fault set as

$$
\mathcal{F}_{\varepsilon}=\left\{K(t)=\operatorname{diag}\left(k_{1}, \ldots, k_{m}\right) \text { satisfying inequality }(21)\right\}
$$

Note the set $\mathcal{F}_{\varepsilon}$ is non-empty since $K=0 \in \mathcal{F}_{\varepsilon}$. In terms of the control law synthesis, $\varepsilon$ is a design parameter.

Theorem 2.1: If the design matrix $M$ has been chosen such that $\hat{A}_{11}(\rho)$ is quadratically stable and $K(t) \in \mathcal{F}_{\epsilon}$, then choosing the modulation gain in (19) to satisfy

$$
\mathcal{K}(t, x)>(1 / \epsilon-1)\left\|v_{l}\right\|+\eta / \epsilon
$$

where $\eta$ is a positive design scalar, ensures a sliding motion takes place on $\mathcal{S}=\{x: S x=0\}$ in finite time.

Proof: See Appendix.

The actual input $u_{p}$ using fixed control allocation is

$$
u_{p}=B_{2}(\rho)^{T}\left(B_{2}(\rho) B_{2}(\rho)^{T}\right)^{-1} B_{v 2}^{T}\left(v_{l}+v_{n}\right)
$$

where $v_{l}$ and $v_{n}$ are defined in (18) and (19). Notice that equation (23) does not contain knowledge of the faults $K(t)$ and so (23) can be implemented without deploying an FDI unit.

\section{MUPAL- $\alpha$ PLATFORM}

MuPAL- $\alpha$, owned and operated by the Japan Aerospace Exploration Agency (JAXA), is a multi-purpose research aircraft used for testing advanced guidance and control technologies and evaluating research on human factors. It is a Dornier Do228-202 aircraft equipped with a research Fly-By-Wire (FBW) system. The MuPAL- $\alpha$ platform (see Fig. 2) supports both HIL tests and actual flight tests for advanced Guidance, Navigation and Control (GNC) technologies (see for example [22], [12], [8], [9], [10]).

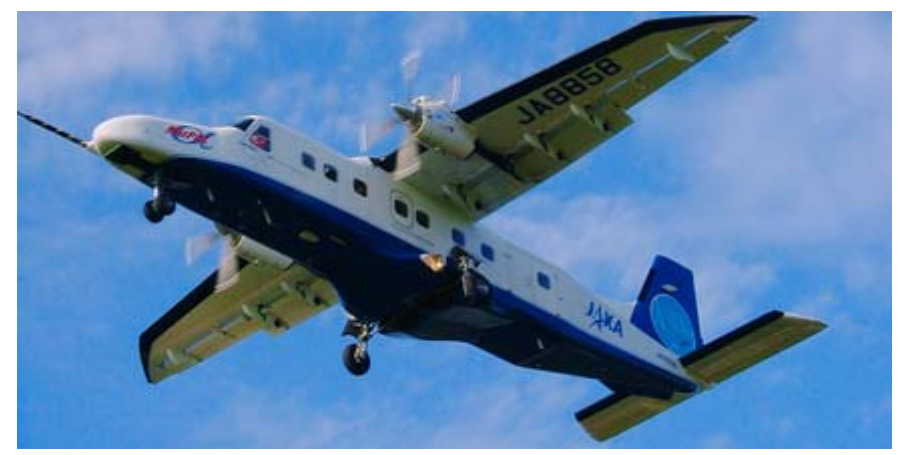

Fig. 2. MuPAL- $\alpha$ aircraft

From a safety aspect, the primary elements of the FBW system are designed to be duplex, which makes it possible to detect system failure by cross-checking the signals. When necessary, the safety pilot can override the FBW and take control through the Do228's mechanical control system [11].

\section{A. LPV modelling}

An LPV model of the lateral dynamics of MuPAL- $\alpha$ has been created in [6], [7] to use as the basis for designing the control law. The scheduling parameters were chosen to be

$$
\rho=\left[\begin{array}{ll}
v_{\text {ias }} & v_{\text {ias }}^{2}
\end{array}\right]
$$

where $v_{\text {ias }}$ denotes the indicated airspeed. In contrast to [23], two scheduling parameters $\left(v_{\text {ias }}\right.$ and $\left.v_{\text {ias }}^{2}\right)$ were chosen to represent the nonlinear dynamics accurately; however, for simplicity, these two parameters are assumed to be mutually independent. Both the scheduling parameters have been normalised to be in the interval $\left[\begin{array}{ll}0 & 1\end{array}\right]$ by appropriate scaling. This paper will focus on lateral-directional control (because compared to the longitudinal axis it has actuator redundancy which can be exploited in terms of fault tolerant control).

The system states are given by

$$
x_{p}=\left[\begin{array}{llll}
\phi & \beta & r & p
\end{array}\right]^{T}
$$

which denote roll angle, sideslip angle, yaw rate and roll rate, respectively. The system inputs $u_{p}$ are given by

$$
u_{p}=\left[\begin{array}{lll}
\delta_{t d} & \delta_{a} & \delta_{r}
\end{array}\right]^{T}
$$




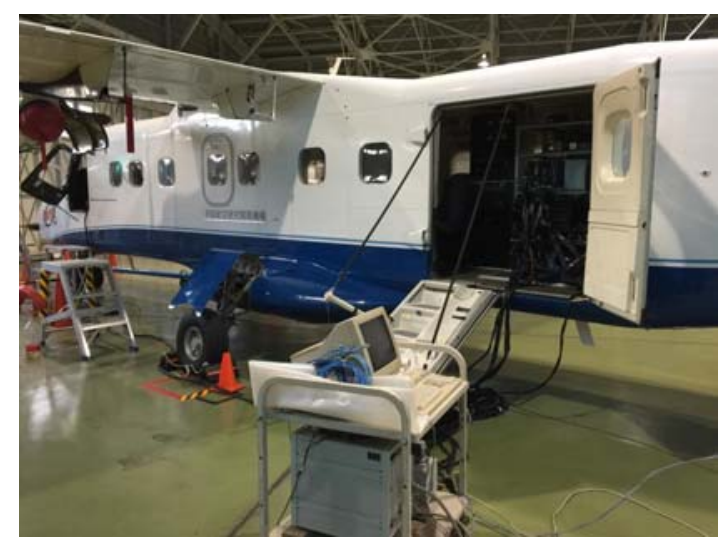

Fig. 3. 'Umbilical cords'

where $\delta_{t d}$ represents differential trust, and $\delta_{a}$ and $\delta_{r}$ represent the aileron and rudder surface deflections.

The controlled outputs are chosen to be

$$
y_{c}=\left[\begin{array}{ll}
\beta & \phi
\end{array}\right]
$$

As argued in [6], under mild assumptions, the input distribution matrix can be approximately factorised as

$$
B(\rho)=B_{v} B_{2}(\rho)
$$

where

$$
B_{v}=\left[\begin{array}{ll}
0 & 0 \\
0 & 0 \\
0 & 0 \\
0 & 0 \\
\hline 1 & 0 \\
0 & 1
\end{array}\right] \quad \text { and } \quad B_{2}(\rho)=\left[\begin{array}{lll}
b_{51}(\rho) & b_{52}(\rho) & b_{53}(\rho) \\
b_{61}(\rho) & b_{62}(\rho) & b_{63}(\rho)
\end{array}\right]
$$

In terms of design, the matrix $M$ has been chosen using a regional 'pole placement' approach [24], [25] to ensure that the 'eigenvalues' of the closed-loop reduced order system associated with $\left(A_{11}(\rho)-A_{12}(\rho) M\right)$, for all fixed values of $\rho$, are to the left of the vertical line through -1 in the complex plane. In (20), the Lyapunov matrix $P_{2}$ and the Hurwitz matrix $\Phi$ are selected as

$$
P_{2}=\left[\begin{array}{ll}
1 & 0 \\
0 & 1
\end{array}\right] \quad \Phi=\left[\begin{array}{cc}
-0.5 & 0 \\
0 & -0.5
\end{array}\right]
$$

For implementation purposes, a 'smooth' approximation of the discontinuous output injection signal from (19) is used [26]: specifically in the implementations

$$
v_{n}=-\mathcal{K}(t, x) \frac{P_{2} s(t)}{\left\|P_{2} s(t)\right\|+\delta}
$$

where the modulation gain $\mathcal{K}=0.4$ and the smoothing factor $\delta=0.05$.

Prior to any flight test, according to JAXA's protocols, any controller must satisfy stringent performance requirements during HIL testing. During the HIL testing, the actual aircraft is employed on the ground with the FBW attached via an umbilical cord (see Fig. 3) to an external PC running a model to simulate the inflight dynamics and provide (virtual) sensor measurements for the Flight Control Computer (FCC). Details of this process are described in [6].

\section{B. Flight tests}

The results presented in the following subsections were obtained from piloted flight tests. The proposed control scheme was initially coded in $\mathrm{C}$ and was integrated into the MuPAL- $\alpha$ FBW system for the HIL test. Once the HIL tests were completed and the results satisfied JAXA's verification and validation requirements, the new control scheme was 'burned' onto the FBW computer.

During the flight tests, the faults have been emulated (at a software level), in the sense that the signal output from the control law is modified (to represent the effect of the fault) before the modified signal is sent to the actuators. 
1) Fault free: One set of fault-free flight test results are shown in Fig. 4. The manoeuvres shown in this paper were created by the evaluation pilot via the pedal and wheel which were subsequently converted into roll and sideslip commands for the controller. During the flight tests, a steady turn with a roll angle of $\pm 20 \mathrm{deg}$ was treated as the reference command. This creates a coordinated ' $\mathrm{S}$ ' turn manoeuvre. The trajectories of the aircraft states associated with the lateral-directional motion are shown in Fig. 4(a). It is clear from Fig. 4(a) that sideslip and roll angle tracking performance are good. Figure 4(b) shows the sliding surfaces and demonstrates that sliding is maintained throughout the flight. The aileron and rudder commands and the actual surface deflections are shown in Fig. 4(c). From Fig. 4(c), it is clear the aileron and the rudder are fault free and the actuator commands do not contain visible chattering.

2) Simultaneous aileron and rudder faults: Figure 5 illustrates the flight test results when faults occur simultaneously on the aileron and the rudder. Here the aileron and the rudder work at $70 \%$ efficiency and $90 \%$ efficiency respectively. This is associated with $K(t)=\operatorname{diag}(1,0.3,0.1)$. It must be stressed however that this information/knowledge is unknown to the controller. The same manoeuvres were repeated in this faulty scenario. The trajectories of the lateral-directional states are shown in Fig. 5(a) wherein again a steady ' $\mathrm{S}$ ' turn manoeuvre with $\pm 20 \mathrm{deg}$ roll angle commands is created by the evaluation pilot. Clearly, although there exist simultaneous rudder and aileron faults, the proposed scheme can still achieve good roll and sideslip tracking performance. Figure 5(b) shows that sliding occurs and the sliding motions are maintained during flight tests. The aileron and rudder commands and their surface deflections are shown in Fig. 5(c). The presence of faults is clearly apparent and the aileron and the rudder cannot follow the respective demands due to the existence of faults.

\section{CONCLUSION}

This paper has developed a sliding mode fixed control allocation scheme and then flight tested it using the Japan Aerospace Exploration Agency's Multi-Purpose Aviation Laboratory (MuPAL- $\alpha$ ) research aircraft. The flight test results show that roll and sideslip tracking performance can be maintained in the presence of simultaneous rudder and aileron faults and environmental conditions.

\section{ACKNOWLEDGEMENTS}

The research leading to these results has received funding from the European Union's Horizon 2020 research and innovation programme under grant agreement No.690811 and the Japan New Energy and Industrial Technology Development Organization under grant agreement No.062800, as part of the EU/Japan joint research project entitled 'Validation of Integrated Safety-enhanced Intelligent flight cONtrol (VISION)'. The work presented here includes contributions from a number of people not directly involved with the preparation of the paper. In particular we gratefully acknowledge the contributions of T. Hosoya, M. Naruoka, J. Kawaguchi, S. Morokuma, H. Ishii and Y. Sagara from JAXA and Y. Uetake from Nakanihon Air Service for their support.

\section{APPENDiX (PROOF OF THEOREM 2.1)}

Multiplying (12) by the matrix $S$ yields

$$
\dot{s}(t)=S A(\rho) x(t)+v(t)+\hat{B}_{c 2} r(t)-B_{v 2} B_{2}(\rho) K(t) B_{2}(\rho)^{\dagger} B_{v 2}^{T} v(t)
$$

Substituting (18) and (19) into (32) yields the reduced order dynamic

$$
\dot{s}(t)=\Phi s(t)-\mathcal{K}(t, x) \frac{P_{2} s(t)}{\left\|P_{2} s(t)\right\|}-B_{v 2} B_{2}(\rho) K(t) B_{2}(\rho)^{T}\left(B_{2}(\rho) B_{2}(\rho)^{T}\right)^{-1} B_{v 2}^{T} v
$$

Now consider a quadratic Lyapunov function given by

$$
V(s)=s^{T} P_{2} s
$$

Differentiating $V(s)$ yields

$$
\dot{V}=s^{T}\left(\Phi^{T} P_{2}+P_{2} \Phi\right) s-2 \mathcal{K}\left\|P_{2} s\right\|-2 s^{T} P_{2} B_{v 2} B_{2}(\rho) K(t) B_{2}(\rho)^{T}\left(B_{2}(\rho) B_{2}(\rho)^{T}\right)^{-1} B_{v 2}^{T} v
$$

Using the fact that $P_{2} \Phi+\Phi^{T} P_{2}=-I_{l}$, it follows

$$
\dot{V}=-\|s\|^{2}-2 \mathcal{K}\left\|P_{2} s\right\|-2 s^{T} P_{2} B_{v 2} B_{2}(\rho) K(t) B_{2}(\rho)^{T}\left(B_{2}(\rho) B_{2}(\rho)^{T}\right)^{-1} B_{v 2}^{T} v
$$

Since $\left\|B_{v 2}\right\|=1$

$$
\left\|B_{v 2} B_{2}(\rho) K(t) B_{2}(\rho)^{T}\left(B_{2}(\rho) B_{2}(\rho)^{T}\right)^{-1} B_{v 2}^{T}\right\| \leq\left\|B_{2}(\rho) K(t) B_{2}(\rho)^{T}\left(B_{2}(\rho) B_{2}(\rho)^{T}\right)^{-1}\right\|
$$

It follows from (21) that

$$
\left\|B_{2}(\rho) K(t) B_{2}(\rho)^{T}\left(B_{2}(\rho) B_{2}(\rho)^{T}\right)^{-1}\right\|<(1-\epsilon)
$$



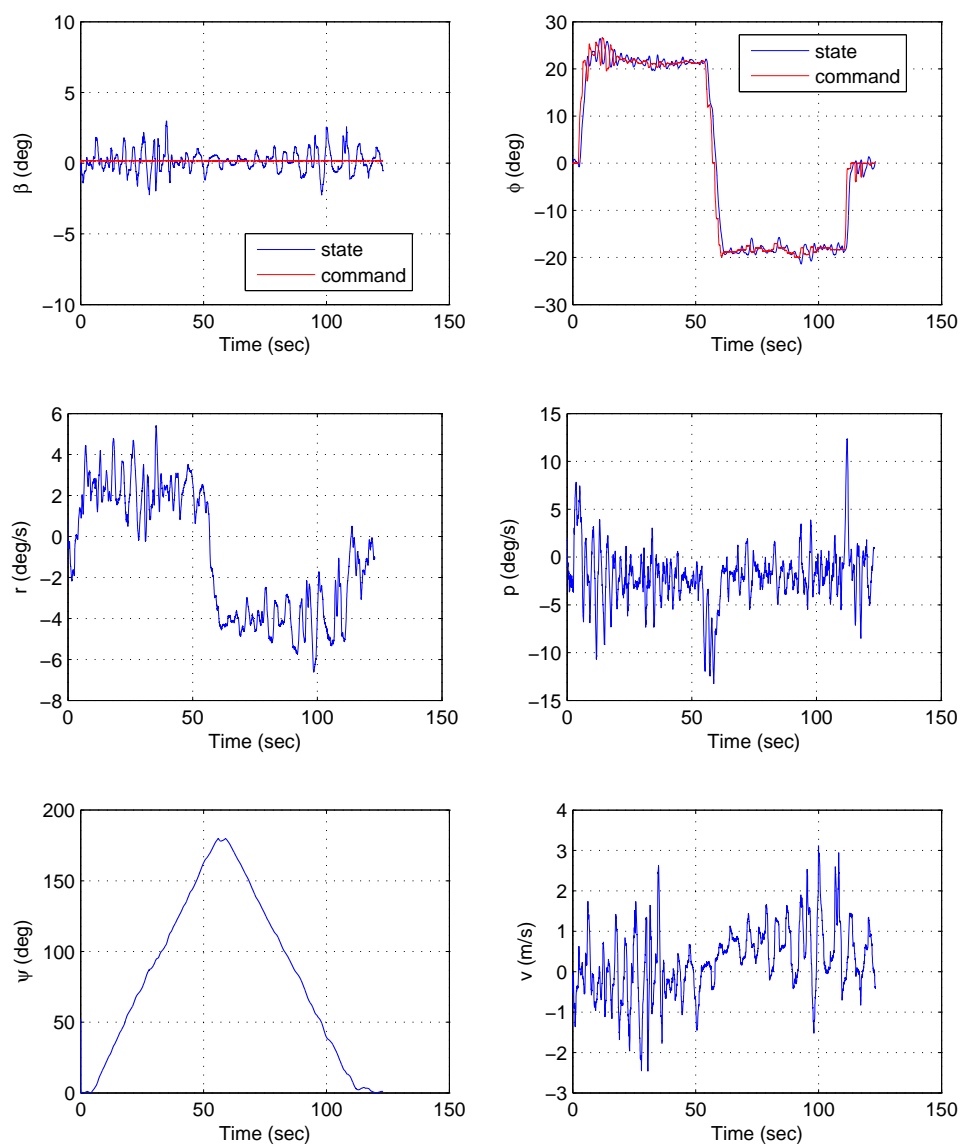

(a) The trajectories of the system states
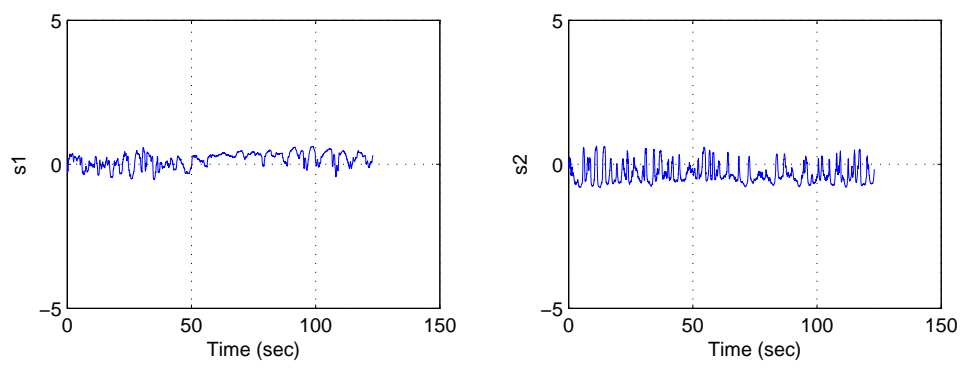

(b) Switching functions
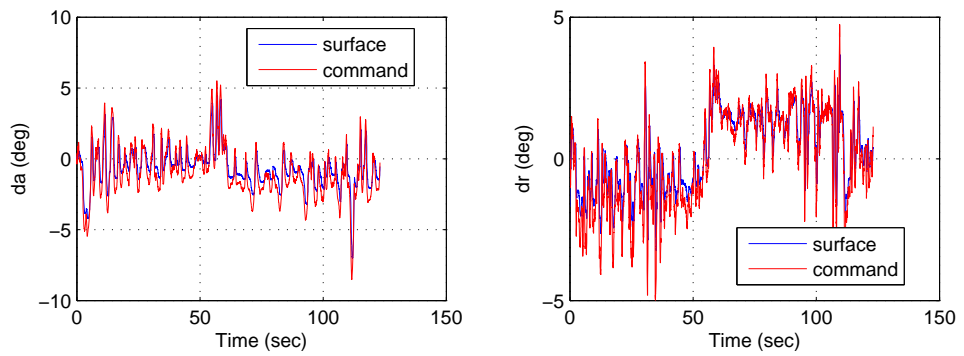

(c) Commands and surface deflections

Fig. 4. Fault-free case: states, switching functions and control surface deflections (Flight test) 

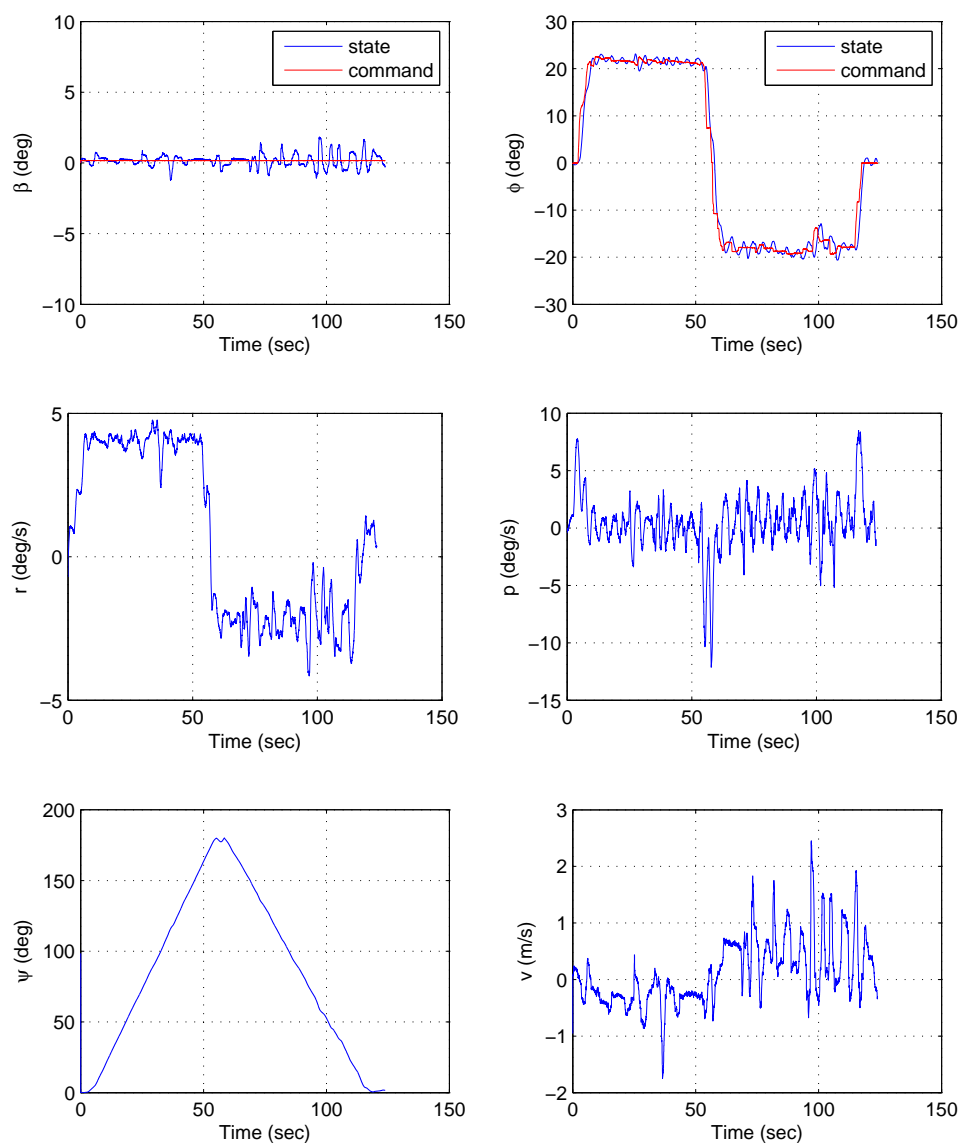

(a) The trajectories of the system states
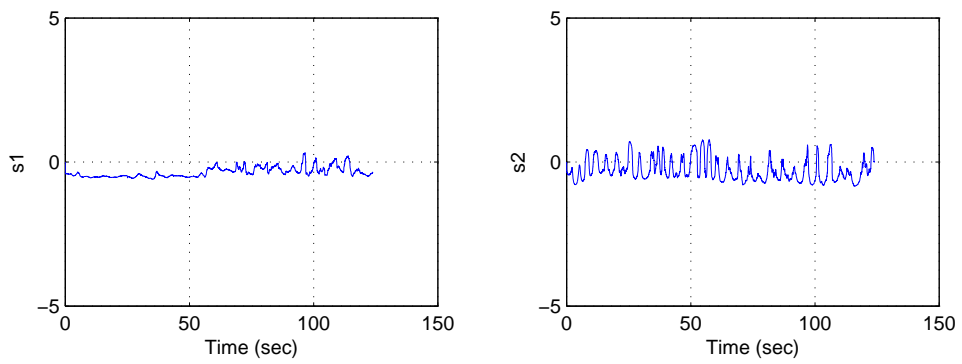

(b) Switching functions
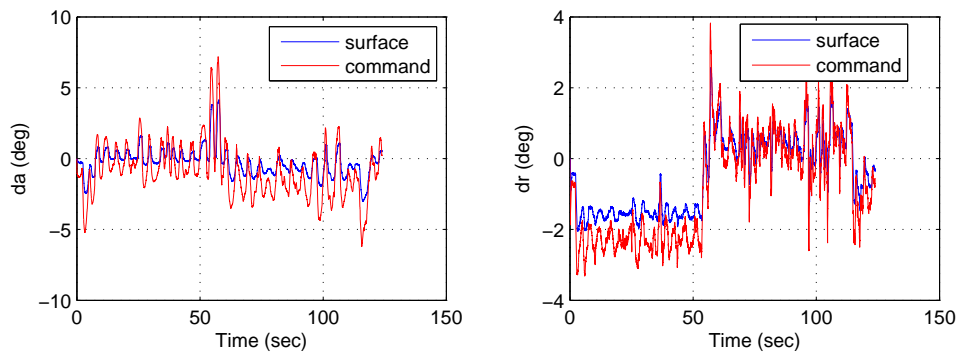

(c) Commands and surface deflections

Fig. 5. Aileron and rudder faults $-K=\operatorname{diag}(1,0.3,0.1)$ : states, switching functions and control surface deflections (Flight test) 
and therefore

$$
\begin{aligned}
\dot{V}(s) & \leq-\|s\|^{2}-2 \mathcal{K}\left\|P_{2} s\right\|+2\left\|P_{2} s\right\|(1-\epsilon)\left(\left\|v_{l}\right\|+\left\|v_{n}\right\|\right) \\
& \leq-\|s\|^{2}-2 \mathcal{K}\left\|P_{2} s\right\|+2\left\|P_{2} s\right\|(1-\epsilon)\left(\left\|v_{l}\right\|+\mathcal{K}(t, x)\right) \\
& \leq-\|s\|^{2}-2\left\|P_{2} s\right\|\left(\epsilon \mathcal{K}-(1-\epsilon)\left\|v_{l}\right\|\right)
\end{aligned}
$$

Substituting (22) into (38) yields

$$
\dot{V}(s) \leq-\|s\|^{2}-2 \eta\left\|P_{2} s\right\|
$$

and then using the Rayleigh principle

$$
\dot{V}(s) \leq-2 \eta \sqrt{V} \sqrt{\lambda_{\min }\left(P_{2}\right)}
$$

It is clear from (40) that the time taken for the trajectory of the closed-loop system to reach the sliding surface $\mathcal{S}$, say $t_{s}$, satisfies

$$
t_{s} \leq \eta^{-1} \frac{\sqrt{V\left(s_{0}\right)}}{\sqrt{\lambda_{\min }\left(P_{2}\right)}}
$$

\section{REFERENCES}

[1] M. Blanke, M. Kinnaert, J. Lunze, and M. Staroswiecki, Diagnosis and Fault-Tolerant Control, 2nd ed. Springer, 2006.

[2] C. Edwards, T. Lombaerts, and H. Smaili, Fault tolerant fight control: A benchmark challenge. Springer, 2010.

[3] P. Goupil and A. Marcos, "The European ADDSAFE project: Industrial and academic efforts towards advanced fault diagnosis," Control Engineering Practice, vol. 31, pp. 109-125, 2014.

[4] P. Goupil, J. Boada-Bauxell, A. Marcos, P. Rosa, M. Kerr, and L. Dalbies, "An overview of the FP7 RECONFIGURE project: Industrial, scientific and technological objectives," in SAFEPROCESS '15, 2015.

[5] P. Goupil, J. Boada-Bauxell, A. Marcos, E. Cortet, M. Kerr, and H. Costa, "AIRBUS efforts toward advanced real-time fault diagnosis and fault tolerant control," in 19th IFAC World Congress, 2014.

[6] L. Chen, H. Alwi, C. Edwards, and M. Sato, "Hardware-in-the-loop evaluation of an LPV sliding mode fixed control allocation scheme on the MuPAL- $\alpha$ research aircraft," in IEEE Conference on Control Technology and Applications, 2017, pp. 590-595.

[7] - "Flight evaluation of an LPV sliding mode controller with online control allocation," in IEEE CDC, 2017.

[8] S. Suzuki and A. Yanagida, "Research and development for fault tolerant flight control system - part 1. intelligent flight control system," in 26th International Congress of the Aeronautical Sciences, 2008.

[9] K. Masui, H. Tomita, and A. Yanagida, "Research and development for fault tolerant flight control system - part 2 flight experiments," in 26th International Congress of the Aeronautical Sciences, 2008.

[10] D. Tokunaga, K. Masui, and S. Suzuki, "Flight evaluation of fault-tolerant control system using simple adaptive control method," in Asia-Pacific International Symposium on Aerospace Technology, APISAT2014, 2014.

[11] K. Masui and Y. Tsukano, "Development of a new in-flight simulator MuPAL- $\alpha, "$ AIAA paper 2000-4574, Aug. 2000.

[12] M. Sato and A. Satoh, "Flight control experiment of multipurpose-aviation-laboratory- $\alpha$ in-flight simulator," Journal of Guidance, Control and Dynamics, vol. 34, 2011.

[13] G. Ferreres and G. Hardier, "Adaptive control of a civil aircraft through on-line parameter estimation," in Systol'16, 2016.

[14] A. Marcos and M. Sato, "Flight testing of an structured H-infinity controller: An EU-Japan collaborative experience," in IEEE CCTA, 2017.

[15] H. Alwi, C. Edwards, and C. P. Tan, Fault Detection and Fault-Tolerant Control Using Sliding Modes. Springer, 2011.

[16] J. Mohammadpour and C. W. Scherer, Control of Linear Parameter Varying Systems with Applications. Springer, 2012.

[17] D. Rotondo, F. Nejjari, and V. Puig, "Robust quasi-LPV model reference FTC of a quadrotor UAV subject to actuator faults," International Journal of Applied Mathematics and Computer Sciences, vol. 25, pp. 7-22, 2015.

[18] H. Alwi and C. Edwards, "Fault tolerant control using sliding modes with on-line control allocation," Automatica, vol. 44, no. 7, pp. 1859-1866, 2008.

[19] T. A. Johansen and T. I. Fossen, "Control allocation - a survey," Automatica, vol. 49, pp. 1087-1103, 2013.

[20] C. Edwards and S. K. Spurgeon, Sliding Mode Control: Theory and Applications. London, U.K.: Taylor \& Francis, 1998.

[21] Y. Shtessel, C. Edwards, L. Fridman, and A. Levant, Sliding Mode Control and Observation. Birkhauser, 2013.

[22] M. Sato and A. Satoh, "Simultaneous realization of handling and gust responses: In-flight simulator controller design," Journal of Guidance, Control and Dynamics, vol. 31, 2008.

[23] M. Sato, "Gain-scheduled flight controller using bounded inexact scheduling parameters," IEEE Transactions on Control Systems Technology, vol. 26, no. 3, pp. 1074-1082, 2018.

[24] M. Chilali and P. Gahinet, " $H_{\infty}$ design with pole placement constraints: An LMI approach," vol. 41, no. 3, 1996.

[25] P. Gahinet, A. Nemirovski, A. Laub, and M. Chilali, LMI Control Toolbox, User Guide. MathWorks, Inc., 1995.

[26] V. Utkin, Sliding modes in control and optimization. Springer, 1992. 